\title{
Les enjeux de la catégorisation
}

Rapports de domination et luttes autour de la représentation dans les sociétés post-migratoires

Marco Martiniello et Patrick Simon

\section{(2) OpenEdition}

12 Journals

Édition électronique

URL : https://journals.openedition.org/remi/2484

DOI : $10.4000 /$ remi.2484

ISSN : $1777-5418$

Éditeur

Université de Poitiers

Édition imprimée

Date de publication : 1 juin 2005

Pagination : 7-18

ISBN : 2-911627-40-7

ISSN : 0765-0752

Référence électronique

Marco Martiniello et Patrick Simon, «Les enjeux de la catégorisation », Revue européenne des

migrations internationales [En ligne], vol. 21 - $\mathrm{n}^{\circ} 2$ | 2005, mis en ligne le 01 octobre 2008, consulté le 14 avril 2022. URL : http://journals.openedition.org/remi/2484 ; DOI : https://doi.org/10.4000/remi.2484

Ce document a été généré automatiquement le 14 avril 2022

(c) Université de Poitiers 


\title{
Les enjeux de la catégorisation
}

\author{
Rapports de domination et luttes autour de la représentation dans les \\ sociétés post-migratoires
}

Marco Martiniello et Patrick Simon

1 Les perspectives constructionnistes (ou constructivistes) nous ont familiarisés avec l'idée que les catégories de représentation et d'action sur la société sont loin d'être immanentes et qu'elles font l'objet d'un travail d'élaboration souvent conflictuel où les capacités d'intervention sont inégalement réparties entre catégorisant et catégorisés. De même, les travaux conduits sur l'histoire de l'Etat se sont attachés à montrer que la faculté de classer, ordonner et administrer les populations comme les biens constituent en soi une des ressources essentielles du pouvoir politique (Foucault, 1976 ; Desrosières, 1993; Noiriel, 2001). Ce faisant, les catégories produites aussi bien par les administrations pour conduire l'action publique que par la statistique pour en évaluer les effets et établir des prévisions ou encore par les sciences sociales pour observer et comprendre la société participent, solidairement, à la formation des identités collectives et à l'encadrement des populations dans des étiquettes sociales correspondant à des rôles et positions institués. Ce qu'Alain Desrosières (1993) qualifie d'encodage met en évidence des opérations de labellisation et de dénomination effectuées par de nombreuses institutions dont la statistique, opérations par lesquelles des «identités » de référence sont énoncées et influencent les propres identifications des acteurs sociaux. En tant que mise en forme du monde social et reconnaissance des groupes pouvant légitimement participer à la vie démocratique et revendiquer éventuellement des droits, la catégorisation constitue un enjeu de pouvoir stratégique dans les sociétés post-migratoires ${ }^{1}$ toutes caractérisées par une multiculturalité importante.

2 Le rôle éminent de l'Etat et de ses institutions dans l'énonciation des identités publiques s'exprime en effet tout particulièrement dans les sociétés multiculturelles où la « politique de l'identité » tend à occuper une place stratégique et recoupe la question des formes de mobilisation sociale et politique des populations « minoritaires », c'est-àdire placées en position subalterne en fonction d'attributs naturalisés. Cynthia Enloe (1981) montre ainsi comment l'Etat intensifie les identités ethniques et joue un rôle 
crucial dans la mobilisation ethnique. Il est un acteur central dans la création, la reproduction et la mobilisation de l'ethnicité à travers la reconnaissance qu'il octroie ou dénie aux groupes ethniques et à travers les processus qu'il met en œuvre en vue de les institutionnaliser (Martiniello, 1995). Les moyens de l'institutionnalisation ne relèvent pas seulement de l'attribution de droits spécifiques ou de l'exercice d'un pouvoir en tant que groupe ; ils commencent par la mise en catégorie et le plus souvent s'y arrêtent. La catégorisation doit s'entendre ici non seulement dans sa dimension technique d'élaboration de catégories administratives ou statistiques par lesquelles les individus sont désignés ou décrits, mais également dans ses dimensions cognitive et pratique par lesquelles les individus sont pensés et perçus. Ainsi, la catégorisation est une activité exercée par de nombreuses instances, dont les pouvoirs d'assignation sont variables, et qui se traduit par une mise en forme plus ou moins contraignante et aboutie des identités collectives ${ }^{2}$.

Cela dit, il faut se garder de tout déterminisme étatique qui simplifierait à outrance le processus de construction identitaire dans lequel les processus de catégorisation et de classification jouent un rôle central. En effet, l'identité se construit dans le rapport à l'autre, au cours de relations qui se tiennent sur plusieurs niveaux : celui des rapports inter-individuels (les contacts sociaux), celui des représentations (confrontation des représentations collectives) et celui du rapport aux institutions de classement (administration et science). Les lieux de la catégorisation et de la classification sont multiples et s'ils ne se construisent pas dans des relations d'équivalence entre catégorisants et catégorisés, les rapports de pouvoir sont loin de s'exercer d'une manière univoque. Une analyse des enjeux de la catégorisation se doit dès lors de distinguer les opérateurs intervenant dans le processus. Parmi les nombreux «marqueurs" susceptibles de fixer "l'emblématique identitaire", les catégories de classement mobilisées par les acteurs sociaux et politiques pour se définir et appréhender les autres (blasonnement social et stéréotypes) forment un objet d'étude particulièrement signifiant. C'est lorsque ces identifications se durcissent, notamment dans les procédures d'enregistrement administratif ou scientifique, par exemple dans la statistique, qu'elles se prêtent le mieux à leur déconstruction. Cependant, d'autres domaines où s'appliquent les taxinomies et se forgent les nomenclatures peuvent servir d'analyseur des formes de gestion de l'altérité et des identités. C'est le cas en particulier de la codification des genres en littérature qu'étudie Alec Hargreaves dans sa contribution à ce dossier. Ici comme pour les identités publiques et les catégories statistiques se repèrent les modes de représentation légitime et la hiérarchisation des productions littéraires ou académiques en genre mineur ou majeur épouse assez fidèlement les grilles mobilisées dans les autres registres de classification.

La thématique identitaire rencontre depuis plusieurs années une vogue croissante, tout en suscitant le scepticisme chez de nombreux chercheurs en sciences sociales, quand ce n'est pas une critique radicale suggérant de l'abandonner en raison de sa conversion en " politiques identitaires » (Brubaker, 2001). C'est bien dans sa connexion à la politique, c'est-à-dire au durcissement des identités dans des identifications retranscrites par des catégories, que la vogue identitaire pose problème et suscite une telle floraison de littérature. Et pourtant, si l'on voit bien les limites et impasses de la politique identitaire, il reste que les sciences sociales auront du mal à se passer de "l'identité» ou à tout le moins doivent construire des outils analytiques permettant de traiter du registre des identités sans tomber dans les généralisations dénoncées par Brubaker. Revenant sur la vogue pour «l'identité culturelle» et les revendications à la 
reconnaissance qui commençaient à s'exprimer, Michel Oriol soulignait le caractère « indispensable et suspect » de la notion. Il ajoutait alors : «On voit mal comment s'en priver s'il s'agit de rendre compte des subversions collectives de la logique des Etats, qui sont rarement, aujourd'hui, du domaine d'un pur et simple affrontement de classe » (1979 : 20). Concept fourre-tout, l'identité et son expression concrète - l'identification - (nous reviendrons plus loin sur cette distinction également développée par Brubaker) constitue un des enjeux majeurs des luttes actuelles pour se doter des moyens de la représentation et obtenir la reconnaissance (Fraser, 2005). Or si la représentation constitue clairement une question centrale pour les sciences sociales (Noiriel, 2001), que ce soit sous l'angle de l'objectivation des faits sociaux, celui de la restitution des expériences et, in fine, de l'interprétation à donner aux processus observés, elle entre explicitement dans le registre du politique. En ce sens, les catégories forgées par les chercheurs sont non seulement en prise avec la doxa politique - et particulièrement tout ce qui relève de «l'immigration » comme le relève Sayad (1999) - mais constituent inséparablement des leviers pour transformer les cadres sociaux et des auxiliaires à l'imposition des normes. Aussi les sciences sociales doivent suivre un étroit chemin de crête entre d'une part la prise au sérieux des effets déterminants de la «culture » et des « identités " (Lamont, 1999) et d'autre part tenir à distance les registres culturalistes et la réutilisation aveugle des catégories du sens commun ou, pire, du politique pour constituer et analyser ses objets.

\section{De la responsabilité des sciences sociales}

5 La réflexion sur les modes de catégorisation a une longue et riche histoire en sciences sociales et il serait déplacé de prétendre que la question est récente ou peu traitée. La responsabilité particulière des chercheurs en sciences sociales, observateurs et performateurs du monde social, est explicitement posée à travers la problématique de la classification. Il est indispensable que les chercheurs s'interrogent sur leurs propres pratiques de catégorisation et sur leurs implications dans les champs médiatique et politique au-delà donc de la sphère académique. Ce questionnement, ou cette prise, par les chercheurs, de leurs pratiques de catégorisation comme objet d'étude, renvoie à différentes problématiques parmi lesquelles celle de l'importation de concepts et catégories d'analyse construites dans d'autres sociétés.

6 Un premier axe d'analyse comparative consiste à observer les effets d'un transfert de politiques ou d'outils catégoriels d'un pays à un autre ou à un ensemble de pays. C'est ce que font Dirk Jabobs et Andrea Rea, dans leur contribution à ce dossier, sur le cas de la migration problématique de la notion d'« allochtone » des Pays-Bas vers la Flandre et progressivement vers la Belgique francophone. Cette transposition s'inscrit plus dans l'extension d'un modèle de "gouvernementalité " que dans celle de la circulation d'idées, concepts et travaux dans le monde académique. Mais elle se légitime également par un mode de pensée qui reproduit progressivement dans ses cadres analytiques les catégories pré-construites par l'administration ou la statistique.

7 Un second axe d'analyse comparative vise à repérer les emprunts conceptuels et leur acclimatation dans d'autres contextes, soit historique, soit sociaux et nationaux. C'est en particulier entre l'Amérique du Nord, et singulièrement les Etats-Unis, et l'Europe que la circulation inégale des théories, concepts et catégories est pointée par les chercheurs visant l'usage a-critique des catégories de «race» ou d'ethnicité. 
Curieusement, le comparatisme, loué par ailleurs, semble trouver ici ses limites. Dans La France de l'intégration (1991: 88) Dominique Schnapper considère que l'appareil conceptuel des "race and ethnic studies » forgé aux Etats-Unis ne peut s'appliquer dans le cas français en raison des différences nationales, confondant les conditions de production des concepts et celle de leur application. S'inscrivant dans une autre tradition critique, Loïc Wacquant (1997) stigmatise l'hégémonie et l'impérialisme de la pensée raciale étatsunienne qui tend à se diffuser dans l'observation et l'analyse des sociétés européennes ou sud-américaines. Rappelant que le trope "racial " résulte d'une configuration historique particulière et que les sciences sociales qui utilisent abondamment les catégories ethniques et raciales se montrent prisonnières du "dilemme américain", Wacquant s'inquiète de la récupération sans recul de ces catégorisations et du substrat théorique qu'elles transportent ${ }^{3}$. Cependant, les modalités de circulation de ces approches conceptuelles et théoriques restent à entreprendre, sur le mode des théories de sciences politiques du framing. Le succès relatif des "ethnic and racial studies" - leur "globalisation" - tient sans doute en partie à des effets de mode et d'imitation pour engranger un capital sur le marché académique international, mais il correspond également à une convergence des configurations sociales et donc des outils forgés pour les décrire. Confrontés aux répliques à distance de la décolonisation et de l'éclatement des anciens empires ou États-nations pluriculturels, d'une part, et à l'affaiblissement des modèles d'intégration verticaux dans le contexte de la globalisation économique d'autre part, les systèmes de gestion politique déploient des ressources similaires pour réguler les hiérarchies ethno-raciales et leurs inévitables tensions (Olzak, 1998). En d'autres termes, les sources de division actives dans les sociétés post-coloniales et post-migratoires tendent à s'homogénéiser et sont traversées par une logique de stratification suivant la fameuse triade : «Classe, genre, race ». Comment dès lors rendre intelligible les évolutions en cours sans restituer ces divisions? Mais comment par ailleurs mobiliser ces catégories de classe, genre et «race» sans valider et renforcer les stéréotypes et logiques de stigmatisation qui sont à l'origine de ces classements?

Beaucoup de travaux ont abordé la question de la responsabilité des chercheurs dans la propagation des stéréotypes forgés par le sens commun ou la validation des catégories réifiantes (Brans et al., 2004). Noiriel (2001), De Rudder (1997) ou Brubaker (2001) insistent tous sur le rôle joué par les sciences sociales dans l'institutionnalisation des identités par le biais de leurs catégories analytiques, souvent trop proches des catégories pratiques. Le problème est que s'ils pointent les abus et usages a-critiques des catégories référant à l'identité, ils ne proposent comme alternative qu'un travail sur les formations des frontières ou sur les identifications. Bien que ce programme de travail constitue l'un des axes de développement de la recherche les plus féconds, il laisse pendante la question des catégories descriptives du monde social. Or on voit mal ne travailler que sur la formation des groupes, sans éléments permettant d'analyser la position d'individus dans l'espace social, ni leurs trajectoires et encore moins les mécanismes par lesquelles les inégalités s'incarnent et sont reproduites. Car pour mettre en évidence des écarts ou des différentiels et leur chercher des explications, encore faut-il délimiter au moins temporairement des catégories d'individus dont les propriétés sociales seront comparées. Cette opération réclame nécessairement de valider des frontières sociales telles qu'elles apparaissent pertinentes à un moment donné de la structuration de la société, et même si ces frontières sont destinées à asseoir une stratification (ce qu'elles font toutes, car toute différenciation ouvre à une forme de 
hiérarchisation ${ }^{4}$ ). Fixer le sujet pour construire l'objet constitue l'acte fondateur de l'analyse en sciences sociales, même si cela expose à réduire le réel et singulièrement à l'enfermer dans un nombre limité de catégories destinées à faire surgir du sens.

Mais cela doit également s'accompagner d'un travail constant de déconstruction des processus d'identification qui participent de manière inséparable à la production des positions et aux critères de distinction. Comme le développe longuement Brubaker, il importe de lever la confusion entre identité et identification, car si la première est proprement insaisissable et donc dépourvue de consistance permettant de l'objectiver par les méthodes ordinaires des sciences sociales, l'autre est justiciable d'un protocole d'observation. Il faut envisager un triangle entre d'une part la condition, c'est-à-dire la position et l'expérience sociales des individus, l'identité individuelle et l'identification, chacun des sommets du triangle interagissant avec les autres : l'identification contribue à façonner l'identité à travers la labellisation sociale ou institutionnelle, la réputation, les représentations...; la condition joue sur l'identification par les ressources mobilisables, la distinction et l'étiquetage social ; l'identité innerve la condition par les dispositions qu'elle façonne et l'identification en proposant des signes interprétés par les « autres » pour classer et labelliser. Cette circularité entre les pôles du triangle rend délicate l'opération de démêlage des relations et interdépendances. De fait, la recherche sur la catégorisation statistique montre que la diffusion de nomenclatures se fait au prix d'un véritable processus d'inculcation et d'intériorisation de la part des enquêtés qui apprennent à se ranger dans les catégories. En ce sens, il n'existe pas "d'auto-déclaration » de soi indépendante des cadres collectifs et des modes légitimes de se présenter, dont le formulaire du questionnaire se fait le vecteur.

10 Enfin, le processus suivi par la production identitaire est étroitement dépendant des contextes historiques, sociaux et politiques dans lesquels il s'inscrit. Ces contextes favorisent les choix et options, ou les imposent, parmi une grande pluralité d'identités à la disposition des individus. La tension entre les options et les contraintes ou assignations forme du reste l'un des objets d'analyse les plus féconds pour comprendre les mécanismes de la production identitaire. Il faut retourner à ce contexte pour réellement saisir l'émergence de concepts et de catégories comme par exemple, la catégorie de Musulmans dans le débat sur l'immigration et l'intégration (voir l'article de Stefano Allievi dans ce dossier).

\section{Mobilisations minoritaires et reconnaissance}

11 Parce que les politiques de l'identité ont longtemps été menées dans le but de dominer, d'opprimer, d'exclure ou d'éradiquer des groupes construits comme inférieurs - et pour certaines ce but est encore d'actualité - la plupart des travaux s'attelant à déconstruire les classifications mettent en évidence les effets d'imposition par les dominants sur les dominés. Pourtant, si l'identification se construit dans des rapports d'inégalité et de domination, elle comporte également un potentiel de subversion qui relativise les déterminations attachées aux assignations catégorielles. Les luttes pour la reconnaissance ne doivent pas uniquement se concevoir sur le plan d'un accès symbolique à la représentation, mais s'inscrivent également dans des mouvements ayant pour objet le contrôle des moyens et formes de la redistribution comme l'a théorisé Nancy Fraser (2005). Au cœur des revendications à la reconnaissance figure la nécessité de requalifier des identités discréditées et donc, pour les minorisés, de se 
réapproprier les catégories forgées dans le rapport de domination pour en modifier le contenu et les signes.

Il nous faut alors approcher la question de l'identification à travers celle du bricolage en déclinant une série d'oppositions: identité imposée/identité revendiquée, reproduction/invention, stigmatisation/affirmation, occultation/reconnaissance et reprendre en détail le jeu auquel se livrent les acteurs, minorisés ou pas, avec les labels les caractérisant. La grammaire identitaire n'est sans doute pas extensible, mais les frontières peuvent être déplacées, en dépit du caractère ontologiquement stigmatisant de la catégorie. Le minoritaire n'est pas uniquement agi, subissant passivement les mécanismes d'imposition identitaire conduits par l'Etat ou le majoritaire et n'ayant comme alternative que le refus des identités prescrites. Christopher Bail et Michèle Lamont reviennent dans ce dossier sur l'enjeu des luttes pour la reconnaissance conduites par les groupes minorisés dans différents contextes et insistent sur le caractère dialectique du processus de catégorisation. Le minoritaire est aussi un acteur central dans la catégorisation, la classification et partant la construction identitaire. Dans certains contextes locaux où le minoritaire devient en fait majoritaire, il peut catégoriser le majoritaire et rendre cette catégorisation socialement inévitable. La catégorisation s'inscrivant donc dans une relation de pouvoir, il ne faut jamais négliger la prise en compte du " pouvoir du faible ».

Or, l'effet de la position subalterne du minoritaire sur sa construction identitaire est inséparablement double: l'étiquetage social le pousse soit à reprendre l'identité stigmatisée pour se l'approprier et la requalifier en inversant le stigmate, selon le processus topique du "black is beautiful », avec le projet ultime de détacher l'image négative de la catégorie; soit à l'inverse à rechercher l'assimilation au groupe dominant ou du moins à son système de signes pour éviter d'être pris dans la dépréciation, avec l'espoir de passer inaperçu et d'éviter les assignations identitaires et le renvoi aux origines. Dans le premier cas, l'effet pervers de la stratégie a été bien étudié: l'investissement dans l'identité stigmatisée tend à renforcer les divisions instituées et sert, au moins dans un premier temps, les intérêts du groupe dominant. Cependant, les minoritaires poursuivant une stratégie d'inversion du stigmate gagnent sur deux points : d'une part ils rendent concret le système de hiérarchisation ethnicoracial ou sexué souvent masqué derrière l'édifice égalitaire formel, ce qui permet d'actualiser l'état réel de la stratification sociale et des modes d'accès aux droits et ressources et d'exercice du pouvoir dans les sociétés multiculturelles. D'autre part, sur le moyen terme, ils construisent les conditions d'un dépassement de la logique identitaire en revenant sur les racines de l'étiquetage par l'appropriation de sa traduction. Celui-ci n'est plus le résultat d'une imposition, ipso facto il change de nature et perd de sa portée. Enfin, les statistiques produites sur la base des catégories résultant des rapports de domination représentent l'une des ressources principales pour faire apparaître les discriminations et alimenter les politiques chargées de les réduire (Simon, 2005). Les mobilisations autour de la classification statistique constituent également un des modes d'expression des luttes pour la reconnaissance. Plutôt que de rejeter les statistiques "raciales", Ludi Simpson recommande au contraire aux mouvements antiracistes de s'emparer de l'outil statistique et de l'exploiter pour démolir la stratification raciale, notamment par le «statistical modelling of institutionnal racism » (Simpson, 2002). 
14 Il n'existe pas de consensus parmi la communauté scientifique et peu de travaux fournissent des éléments permettant d'envisager les "coûts et bénéfices» de la reconnaissance et de la banalisation des topiques ethniques et raciales. Les théories qui rapportent la visibilisation des « différences » aux rapports de domination, c'est-à-dire qui postulent la non-existence intrinsèque de différences, et donc de "groupes ethniques » ou "raciaux », avant que ne s'engage un rapport raciste ou "ethniste » fournissent une source précieuse d'intelligibilité de la formation des groupes et des catégories, mais elles n'expliquent pas comment faire sans ces catégories. Le réinvestissement positif d'une identité qui est ontologiquement générée à des fins de subordination n'est en substance pas concevable et donc toute lutte pour la reconnaissance ne peut que constituer un renforcement de l'oppression initiale ${ }^{5}$. Dans ce paradigme d'analyse, la perspective d'inversion du stigmate n'est évidemment pas ignorée, mais elle est globalement conçue comme vaine et condamnée à terme. Le renforcement de la différenciation étant le prix à payer, toute stratégie de reconnaissance comporterait ontologiquement les conditions de son échec politique.

Difficile d'interpréter leurs positions à l'égard de leur identification sans commencer par rappeler dans quel cadre de référence national et social ils évoluent. Ici, les écarts entre les pays dits multiculturalistes et les pays dits assimilationnistes produisent des effets déterminants, et on n'observera pas les mêmes formes de revendication et d'investissement dans des identités collectives à New York, Berlin, Amsterdam, Londres ou Paris. Les tropismes nationaux et locaux construisent les conditions de l'identification et le type de position adoptée à l'égard de la classification : la recherche d'une invisibilisation ou l'affirmation d'une appartenance en dépendent étroitement. L'une des grandes réussites du modèle français d'intégration, selon Sayad, est d'avoir renforcé les conduites "d'hypercorrection sociale », amenant à accepter « la définition dominante de son identité » et à rechercher l'assimilation en développant «tout un travail de présentation de soi et de représentation, donc un travail portant essentiellement sur le corps, sur l'apparence physique, sur les comportements extérieurs le plus chargés précisément d'attributs ou de significations symboliques, afin, d'une part de faire disparaître tous les signes susceptibles de rappeler le stigmate [...] et, d'autre part, d'afficher par mimétisme l'adoption de traits qui, par contraste, semblent être caractéristiques emblématiquement de ceux auxquels on voudrait s'assimiler.» (Sayad, 1999: 10). Même si l'observation de Sayad ne semble plus correspondre parfaitement à la réalité urbaine française, elle reste historiquement correcte. Nacira Guénif revient dans ce dossier sur le contexte créé par les injonctions du modèle d'intégration français et ses conséquences sur la formation des identités sexuées des descendants de l'immigration post-coloniale. Pris entre le désir d'accéder enfin aux dividendes du "modèle» et cherchant à se réinscrire dans l'histoire familiale, peu représentée dans le roman national, les descendants d'immigrés entretiennent des rapports ambivalents avec la reconnaissance.

16 À l'opposé des contextes assimilationnistes poussant à rechercher l'invisibilité, les pays dits multiculturalistes se caractérisent par la profusion de références et de signes exprimant et valorisant la "différence " (Taylor, 1992). L'expérience des pays ayant développé des catégorisations faisant référence à l'ethnicité ou à la "race » met en évidence le rôle de groupes de pression-lobbies portant la parole des minorités et se trouvant en situation d'infléchir les décisions politico-administratives. De nombreux cas sont évoqués par Kertzer et Arel dans leur analyse des relations entre les 
recensements et le pouvoir (2002: 27-31). La première condition de réussite est que les groupes soient dotés de porte-parole ou du moins d'un espace d'intervention leur permettant d'entrer en médiation. Or de telles structures de mobilisation collective n'existent pas dans de nombreux pays où les cadres politiques rendent sinon impossibles, du moins illégitimes, les plateformes d'action sur une base ethnico-raciale. Dans ces conditions d'absence d'expression politique venant des minorités, les sciences sociales se retrouvent en situation d'interprètes des expériences et, par là, des projets des minorisés. On comprend dès lors que dans les pays où les lobbies minoritaires n'existent pas, au premier rang desquels figure la France, les débats sur les catégorisations scientifiques et la mise en forme des résultats des observations prennent une dimension passionnelle. Plus qu'une simple question méthodologique, la classification prend une portée strictement politique qui s'exprime de manière plus évidente que dans les autres contextes.

17 Chaque société produit ses propres cadres de classification et des catégories en apparence similaires peuvent fort bien résulter de processus historiques distincts, servir des objectifs diamétralement opposés et recevoir des significations extrêmement différentes (Rallu et al., 2004). Il importe dès lors de replacer l'opération de catégorisation dans son contexte d'élaboration et son cadre d'utilisation pour éviter de plaquer des significations décalées. C'est le cas par exemple des catégories raciales aux Etats-Unis qui se présentent comme l'exact équivalent de celles forgées après l'abolition de l'esclavage et dans un contexte de ségrégation raciale et de discrimination officielle, mais qui servent désormais des objectifs de promotion de l'égalité et de réparation des torts historiques (affirmative action) (Martiniello et Rea, 2003). L'illusion d'une continuité des fins de la politique en raison de la permanence du système de catégorisation ne tient pas face à la rupture engagée par le mouvement des droits civiques. On ne saurait dès lors interpréter la classification raciale dans la statistique étatsunienne de la même façon en 1930 et en 2000. Ann Morning revient dans ce dossier sur l'introduction dans le recensement étatsunien de 2000 de la possibilité de se déclarer plus d'une "race» et montre comment la représentation d'une Amérique multiraciale peut tout aussi bien miner de l'intérieur l'édifice du racialisme que favoriser sa recomposition sous le vernis de la colorblindness. Si les EtatsUnis se caractérisent par l'omniprésence des catégories ethnico-raciales pour penser et agir sur la société, d'autres pays dont la France se distinguent par une sorte de colorblindness revendiquée et poussée à ses extrêmes. Du point de vue des mécanismes de la domination, l'absence de reconnaissance constitue également un outil particulièrement efficace. L'invisibilité empêche les minorisés de participer aux prises de décisions politiques et contribue au déni de l'expérience des discriminations. Plus encore, l'apparente " indifférence aux différences » masque une politique des identités qui fonctionne en creux, en promouvant l'identité du majoritaire comme une référence à imiter pour pouvoir entrer pleinement dans la citoyenneté (Mouvements, 2005).

Ces différentes interprétations des évolutions en cours nous renvoient à la genèse même de la construction des catégories raciales et aux conditions de leur utilisation. En tant que telles, il ne fait pas de doute qu'elles trouvent leur origine dans le système de domination raciale construit lors de l'expansionnisme européen et concrétisé d'une part dans l'histoire de l'esclavage et de la colonisation et d'autre part dans la formation de la pensée racialiste au cours de la deuxième moitié du XIX siècle (Guillaumin, 1972). Ces origines sont réactivées, sous des formes retravaillées, dans les rapports de subordination qui sont le propre des sociétés hiérarchisées. Faut-il pour autant suivre 
C. Delphy lorsqu'elle pose que toute différence est rattachée à un ordre hiérarchique et qu'elle n'existe donc pas en dehors de cette visée de domination (2001: 9 et suiv.) ? Argument développé également par V. de Rudder et al. qui le résument en une formule définitive : "toute classification raciale est, par définition, une classification raciste. " (2000 : 35). Dans ces conditions, comment penser les marges de manœuvre autour de la catégorisation? Comment parler du racisme et comment analyser les discriminations? L'interrogation vaut bien entendu pour les observateurs de la société que sont les chercheurs en sciences sociales. Mais elle s'étend également aux minorités qui « héritent » des statuts et catégories assignés : la lutte pour la reconnaissance n'est-elle qu'une défaite programmée?

\section{BIBLIOGRAPHIE}

BARRERE Jean-Yves (2000) Classer les exclus : enjeux d'une doctrine de politique sociale, Paris, Dunod.

BOLTANSKI Luc (1982) Les cadres. La formation d'un groupe social, Paris, Ed. de Minuit.

BOURDIEU Pierre (1980) L'identité et la représentation. Eléments pour une réflexion critique sur l'idée de région, Actes de la recherche en sciences sociales, $\mathrm{n}^{\circ}$ 35, pp. 63-72.

BRANS Marleen et al. (2004) Recherches et Politiques publiques : le cas de l'immigration en Belgique, Gand, Academia Press, Série « science et société ».

BRUBAKER Rogers (2001) Au-delà de « l'identité », Actes de la Recherche en Sciences Sociales, 139, pp. 66-85.

CASTLES Stephen, BOOTH Heather and WALLACE Tina (1984) Here for Good. Western Europe's New Ethnic Minorities, London : Pluto Press

DELPHY Christine (2001) Critique de la raison naturelle, Préface in L'ennemi principal (tome II « Penser le genre »), Paris, Syllepse, pp. 7-53.

DE RUDDER Véronique (1997) Quelques problèmes épistémologiques liés aux définitions des populations immigrantes et de leur descendance, in Aubert France. Tripier Maryse et Vourc'h François, Jeunes issus de l'immigration, de l'école à l'emploi, Paris, L'Harmattan.

DE RUDDER Véronique, POIRET Christian et VOURC'H François (2000) L'inégalité raciste.

L'universalité républicaine à l'épreuve, Paris, PUF.

DESROSIERES Alain (1993) La politique des grands nombres. Histoire de la raison statistique, Paris, La Découverte.

DUMONT Louis (1979) Homo Hierarchicus ; le système des castes et ses implications, Paris, Gallimard.

DUMONT Louis (1983) Essais sur l'individualisme. Une perspective anthropologique sur l'idéologie moderne, Paris, Seuil.

DURKHEIM Emile et MAUSS Marcel (1903) De quelques formes de classification - contribution à l'étude des représentations collectives, Année Sociologique, $\mathrm{n}^{\circ} 6$ (republié in M. Mauss, Essais de sociologie, Point-Seuil, 1971). 
ENLOE Cynthia (1981) The growth of the state and ethnic mobilization: the American experience, Ethnic and Racial Studies, 4, 2, pp. 123-136

FOUCAULT Michel (1976) La volonté de savoir, Histoire de la sexualité, tome I, Paris, Gallimard.

FRASER Nancy (2005) Qu'est-ce que la justice sociale ? Reconnaissance et redistribution, Paris, La Découverte.

GUILLAUMIN Colette (1972) L'idéologie raciste : genèse et langage actuel, Paris, La Haye, Mouton.

KERTZER David, AREL Dominique (2002) Censuses, identity formation, and the struggle for political power, in Census and identity. The politics of race, ethnicity and language in national censuses, Kertzer and Arel (Ed), Cambridge, Cambridge University Press, pp. 1-42.

LAMONT Michèle éd. (1999) The cultural territories of race : black and white boundaries, Chicago, University of Chicago Press ; New York : Russell Sage Foundation.

MARTINIELLO Marco (1993) Pour une sociologie politique de la situation post-migratoire en Belgique, in Martiniello M. et Poncelet M. (sous la direction de), Migrations et minorités ethniques dans l'espace européen, Bruxelles : De Boeck Université, L'homme/L'étranger, pp. 167-185.

MARTINIELLO Marco (1995) L'ethnicité dans les sciences sociales contemporaines, Paris : P.U.F., «Que sais-je? », n² 2997.

MARTINIELLO Marco (1996) Ethnicité, « race » et classe sociale : le débat au sujet de l'underclass aux États-Unis et en Europe, La Revue Tocqueville, 17 h 2, pp. 117-134.

MARTINIELLO Marco et REA Andrea éds. (2003) Affirmative Action. Des discours, des politiques et des pratiques en débat, Louvain-La-Neuve, Academia-Bruylant.

Mouvements (2005) La politique républicaine de l'identité, numéro 38, avril-mai, La Découverte. NOIRIEL Gérard (1997) Représentation nationale et catégories sociales. L'exemple des réfugiés politiques, Genèses, 26, pp. 25-54.

NOIRIEL Gérard (2001) État, nation et immigration. Vers une histoire du pouvoir, Paris, Belin.

OLZAK Susan (1998) Ethnic protest in core and periphery state, Ethnic and Racial Studies, 21-2, pp. 187-217.

ORIOL Michel (1979) Identité produite, identité instituée, identité exprimée : confusion des théories de l'identité nationale et culturelle, Cahiers Internationaux de Sociologie, vol 66, pp. 19-28.

RALLU Jean-Louis, PICHÉ Victor et SIMON Patrick (2004) Démographie et ethnicité : une relation ambiguë, in Démographie : analyse et synthèse (Vol VI : Population et société), Caselli G., Vallin J. et Wunsch G. éd., Paris, INED-PUF, pp.481-516.

RINAUDO Christian (1998) L'imputation de caractéristiques ethniques dans l'encadrement de la vie scolaire, Revue Européenne des Migrations Internationales, 14-3, pp. 27-43.

SAYAD Abdelmalek (1999) Immigration et " pensée d'état », Actes de la Recherche en Sciences Sociales, $\mathrm{n}^{\circ} 129$, pp. 5-14.

SCHNAPPER Dominique (1991) La France de l'intégration, Sociologie de la Nation en 1990, Paris, Gallimard.

SIMON Patrick (2003) Le logement social en France et la gestion des " populations à risque ", Hommes et Migrations, $\mathrm{n}^{\circ}$ 1246, pp. 78-91.

SIMON Patrick (2005) The measurement of racial discrimination: the policy use of statistics, International Journal of Social Science, $\mathrm{n}^{\circ}$ 183, pp. 9-25. 
SIMPSON Ludi (2002) Race statistics : Their's and Our's, Radical Statistics, 79/80.

http://www.radstats.org.uk/no079/simpson.htm (consulté 09/2005)

TAYLOR Charles (1992) Multiculturalism and « the politics of recognition », Princeton, Princeton University Press (trd. 1994, Multiculturalisme, différence et démocratie, Paris, Aubier).

WACQUANT Loïc (1997) For an analytic of racial domination, Political power and social theory, 11.

WACQUANT Loïc (2005) De l'esclavage à l'incarcération de masse. Notes pour repenser la « question noire » aux Etats-Unis, in Weil Patrick et Dufoix Stéphane dir. L'esclavage, la colonisation, et après..., Paris, PUF, pp. 247-273.

\section{NOTES}

1. La plupart des pays de l'Union Européenne connaissent à la fois une situation migratoire et une situation post-migratoire (Martiniello, 1993). D'une part, les flux migratoires à destination de l'Europe se développent parfois sous des formes neuves. D'autre part, les vagues migratoires anciennes ont donné lieu à un établissement définitif des immigrés et de leurs descendants qui s'est traduit par la formation de nouvelles minorités ethniques dans l'espace européen en évolution (Castles, Booth et Wallace, 1984). La situation post-migratoire se caractérise par la cohabitation entre les citoyens sans passé migratoire reconnu (« les autochtones »), les minorités ethniques issues des migrations anciennes et les nouveaux arrivants dont la position juridique et sociale reste souvent la plus précaire.

2. Dans le grand marché de l'assignation des identités, comme le rappellent Kertzer et Arel (2002), les recensements nationaux jouent un rôle déterminant pour la formation des identifications. Celles-ci sont cependant véhiculées dans les interactions quotidiennes avec les instances d'encadrement, telle que l'école (Rinaudo, 1998), le logement social (Simon, 2003), les politiques sociales (Barreyre, 2000).

3. Martiniello (1996) émet une critique analogue de l'usage que fait Lapeyronnie de la notion hautement problématique d'underclass.

4. Point discuté par Louis Dumont (1983) et développé par Christine Delphy dans sa critique du féminisme différentialiste (2001).

5. Relevant que «les revendications d'égalité se transforment en revendication d'identité », C. Delphy y voit une « défaite pour le groupe..., un jeu de dupes» (2001:9-10).

\section{RÉSUMÉS}

En fournissant les cadres de représentation et d'action sur le monde social, la catégorisation constitue un enjeu de pouvoir stratégique dans les sociétés post-migratoires. Les groupes minorisés sont confrontés à un dilemme que reflètent bien les débats dans les sciences sociales : investir les identités collectives stigmatisées qui leur ont été historiquement assignées pour tenter de les requalifier ou s'en détacher pour produire d'autres identifications. Cette question de stratégie politique qui est posée aux minorités s'impose également avec d'autres enjeux pour les sciences sociales: la responsabilité des chercheurs face à la politique des identités et la construction de leurs objets face aux catégorisations forgées pour asseoir des hiérarchies. Ce 
texte balaie les différentes dimensions développées dans ce dossier consacré aux catégorisations et aux rapports de domination.

Categorization at Stake. Domination and Conflicts for Representation in Postmigratory Societies.

Because it provides the framework for representation and action in the social world, categorization is a strategic power stake in postmigratory societies. Minority groups are confronted with a dilemma that reflects debates in the social sciences: invest in stigmatized collective identities that have been historically assigned to them in order either to try to value them or to produce new identifications. The question of the political strategy faces by minorities is also relevant, in other ways for social sciences: the responsibility of researchers faced with the politics of identities and the construction of research objects faced with categorizations built to reproduce hierarchies. This article presents an overview of the various dimensions approached in this issue dealing with categorization and relations of domination.

Categorización y poder. Dominación y conflictos alrededor de la representación en las sociedades postmigratorias.

Porque provee los marcos de representación y de acción en el mundo social, la categorización tiene un poder estratégico en las sociedades postmigratorias. Los grupos minorizados tienen que enfrentar un dilema que los debates en las ciencias sociales reflejan bien: investir las identidades colectivas desvalorizadas que se le han asignado históricamente para tratar de valorizarlas o alejarse de ellas para producir otras identificaciones. Este problema de estrategia política planteado a las minorías se plantea también, de otro modo, a las ciencias sociales: la responsabilidad de los investigadores frente a las políticas de identidades y la construcción de sus temas frente a las categorías forjadas para reproducir las jerarquías existentes. Este texto presenta las diferentes dimensiones desarrolladas en este dossier dedicado a las categorizaciones y a las relaciones de dominación.

INDEX

Mots-clés : identité collective, minorités, représentations

\section{AUTEURS}

\section{MARCO MARTINIELLO}

Directeur de recherches FNRS. Université de Liège. Centre d'Etudes de l'Ethnicité et des Migrations (CEDEM), Institut des Sciences Humaines et Sociales, bâtiment 31, boîte 45, 7 bd du Rectorat, 4000 Liège, Belgique, M.Martinello@ulg.ac.be

\section{PATRICK SIMON}

Socio-démographe, Institut National d'études Démographiques (INED), Unité Migrations Internationales et Minorités, 133 rue Davout, 75020 Paris, France. simon@ined.fr 